\title{
Is there any association of COVID-19 with Testicular Pain and Epididymo-orchitis
}

\author{
Caner Ediz ${ }^{1}$, Hasan Huseyin Tavukcu ${ }^{2}$, Serkan Akan ${ }^{3}$, Yunus Emre Kizilkan ${ }^{2}$, Adem \\ Alcin $^{2}$, Kerem Oz ${ }^{1}$, and Omer Yilmaz ${ }^{2}$ \\ ${ }^{1}$ Sultan Abdulhamid Han Egitim ve Arastirma Hastanesi \\ ${ }^{2}$ Sultan Abdülhamid Han Eğitim ve Araştırma Hastanesi \\ ${ }^{3}$ Sultan Abdülhamid Han Training and Research Hospital
}

September 28, 2020

\begin{abstract}
Aims: This study aims to analyze the novel Coronavirus disease (COVID-19) related testicular pain in hospitalized patients due to COVID-19 and to review as an etiological factor for epididymitis, orchitis or both. Methods: A total of 91 patients were included in the study. A questionnaire was formed for the questioning of testicular pain or epididymo-orchitis in patients with COVID-19. Demophrahics and past medical history was also recorded. Patients' neutrophil and lymphocyte counts, neutrophillymphocyte ratios (NLR), C-reactive protein (CRP) levels and D-dimer values were recorded. Patients with COVID-19 were divided into two groups according to absence or presence of testicular pain or epididymo-orchitis as group 1 and group 2 . All results were compared for both groups. Results: The median age of patients was similar in both groups. Testicular pain was occured in $10.98 \%$ of the patients. Clinical presentation of epididymo-orchitis was diagnosed in only one patient. No statistically significant difference was reported in terms of patients' age, levels of CRP and D-Dimer or NLR and results of questionnaire form queries between the two groups $(\mathrm{p}>0.05)$. Conclusion: Testicular pain was observed more frequently in hospitalized COVID-19 cases. While no inflammation marker which is related to predict of testicular pain or epididymo-orchitis was found in patients with COVID-19.
\end{abstract}

\section{Is there any association of COVID-19 with}

\section{Testicular Pain and Epididymo-orchitis}

Ediz Caner ${ }^{*}$, Tavukcu Hasan Huseyin ${ }^{1 *}$, Akan Serkan ${ }^{1}$, Kizilkan Yunus Emre ${ }^{1}$, Alcin Adem¹ ${ }^{1}$ Oz Kerem ${ }^{1}$, Yilmaz Omer ${ }^{1}$

${ }^{1}$ Department of Urology, Sultan Abdulhamid Han Education and Research Hospital, Istanbul, Turkey

\section{Corresponding Author}

Caner Ediz, MD

Department of Urology

Sultan Abdulhamid Han Education and Research Hospital, Istanbul, Turkey

Postal Address: Tibbiye Cd, Selimiye Mh., Uskudar/Istanbul

Postal Code: 34668

Phone: +90 53358050 80; Fax: +90 2165422020

E-mail:drcanerediz@gmail.com 


\begin{abstract}
Aims: This study aims to analyze the novel Coronavirus disease related testicular pain in hospitalized patients due to COVID-19 and to review as an etiological factor for epididymitis, orchitis or both.
\end{abstract}

Methods: A total of 91 patients were included in the study. A questionnaire was formed for the questioning of testicular pain or epididymo-orchitis in patients with COVID-19. Demophrahics and past medical history was also recorded. Patients' neutrophil and lymphocyte counts, neutrophil-lymphocyte ratios, C-reactive protein levels and D-dimer values were recorded. Patients with COVID-19 were divided into two groups according to absence or presence of testicular pain or epididymo-orchitis as group 1 and group 2. All results were compared for both groups.

Results: The median age of patients was similar in both groups. Testicular pain was occured in 10.98\% of the patients. Clinical presentation of epididymo-orchitis was diagnosed in only one patient. No statistically significant difference was reported in terms of patients' age, levels of CRP and D-Dimer or NLR and results of questionnaire form queries between the two groups .

Conclusion: Testicular pain was observed more frequently in hospitalized COVID-19 cases. While no inflammation marker which is related to predict of testicular pain or epididymo-orchitis was found in patients with COVID-19.

Keywords: Coronavirus disease, COVID-19, epididymitis, epididymo-orchitis, orchitis, testicular pain

\title{
Introduction
}

Coronaviruse cause either self-limiting mild infection or more serious infections that effect to the whole body. CoV disease has first reported on January 7, 2020, as a result of investigations from pneumonia cases of unknown etiology in Wuhan, China's Hubei province. COVID-19 caused a pandemic that has begun as pneumonia of unclear etiology which has spread all over the world. The disease is transmitted mainly through droplets. As the virus can be detected in the respiratory secretions of asymptomatic people, of whome could transmit the disease to another people.

The recommendations of the genitourinary system associated with COVID-19 are generally focused on management of urological diseases or treatment methods. ${ }^{1-4}$ If we evaluated in terms of genitourinary pathologies, acute kidney failure is among the most frequently studied subjects. ${ }^{5,6}$ Studies published of the relationship between the genitourinary cancers and Covid-19 has usually focused on the management of prostate cancer or bladder cancer.$^{7-14}$ In these studies, the roles of $\mathrm{CoV}$ presence in the diagnosis and treatment of urinary system pathologies were mentioned and disease management was explained with general recommendations.

The relationship between testicular pain and COVID-19 is one of the least informed subjects among the existing urological diseases. In the current literature, only one case report was published regarding COVID19 related scrotal or testicular pain. ${ }^{15,16}$ In this study, we aimed to evaluate COVID-19 related testicular pain in hospitalized patients due to COVID-19 and to review as an etiological factor for epididymo-orchitis.

\section{Material Methods}

In this prospective descriptive study, patients who diagnosed and treated due to COVID-19 in our clinic between April 1, 2020 and May 31, 2020 were included. This study was approved by the local Ethics Committee and conducted according to the principles of World Medical Association Declaration of Helsinki 'Ethical Principles for Medical Research Involving Human Subjects.

A total of 91 male patients diagnosed with COVID-19 were enrolled to study. Patients with COVID-19 between 18 and 75 years of were included in the study. Patients who were found to have missing data during data recording, evaluation or analysis were excluded from the study. To evaluate testicular pain or epididymo-orchitis in patients with COVID-19, some questions related with urological complaints such as urinary system symptoms, previous surgical and medical history, or the presence of epididymo-orchitis were assessed in all patients. Patients' neutrophil and lymphocyte count, C-reactive protein levels, D-dimer 
values and neutrophil-lymphocyte ratios were recorded. Patients with COVID-19 were divided into two groups according to absence or presence of testicular pain as group 1 and group 2. 81 patients in group 1 and 10 patients in group 2 were enrolled to the study. All results of questionnaire form and laboratory tests were compared for both groups.

Data analyses were performed using SPSS Statistics 20.0 software. The normality hypothesis was tested using the Kolmogorov-Smirnov test during data analysis. All variables were non-normally distributed. Quantitative variables were expressed as median. Qualitative variables were expressed as presence or absence percentage, and Chi-square test was applied. Mann-Whitney's U-test was used to evaluate all non-normally distributed variables. A $\mathrm{p}<0.05$ was considered statistically significant in all analyses.

\section{Results}

A total of 91 patients were analyzed. Eighty-one and ten patients were enrolled in group 1 and 2, respectively. The median age of the patients in group 1 was 37 years and in group 2 was 46 years. Presence of dysuria, testicular swelling, history of BCG vaccine, history of epididymo-orchitis and any Urological surgery were shown in Table 1. Median duration of COVID-19 was similar in both groups and no statistically significant difference was found. Median neutrophil and lymphocyt counts, NLR, CRP and D-Dimer levels for both groups were summarized in Table 2. No statistically significant difference was found between in two groups for these parameters .

Patients were evaluated according to the level of lymphopenia to find any association of stage of COVID-19 disease with testicular pain or epididymo-orchitis. The patients were divided into two groups as those with lymphocyte level below $1.5 \times 10^{3} / \mathrm{mm}^{3}$ and above $1.5 \times 10^{3} / \mathrm{mm}^{3}$. Groups were compared for testicular pain and epididymo-orchitis. Patients with lymphocyte level below $1.5 \times 10^{3} / \mathrm{mm}^{3}$ were older than the patients with lymphocyte level above $1.5 \times 10^{3} / \mathrm{mm}^{3}$ and it was statistically significant . No statistically significant difference was found in the groups for other parameters and descripted in Table 3.

\section{Discussion}

Testicular pain is divided two groups according to acute or chronic. Acute severe testicular pain is usually related with testicular torsion but chronic testicular pain is a rare condition and be considered as part of the chronic pelvic pain syndrome. ${ }^{17}$ Infectious or non-infectious causes may induce to acute testicular pain. Isolated acute orchitis is an infrequent phenomenon and usually accompanied by epididymitis. ${ }^{18}$ There are nearly 600,000 cases of epididymo-orchitis per year in the United States and the majority of epididymoorchitis cases are aged 20 to 39 years. ${ }^{19}$

Testicular pain and epididymo-orchitis caused by bacterial pathogens specifically Chlamydia trachomatis and Neisseria gonorrhoeae, are a common condition in andrology. Orchitis in especially young patients is caused by virus infections such as mumps, rubella, coxsackievirus, varicella, echovirus, and cytomegalovirus. ${ }^{20,21}$ Since the using of the mumps-measles-rubella vaccination, the frequency of mumps orchitis has decreased dramatically.

Mechanism of occured COVID-19 infection by binding to Angiotensin-converting enzyme 2 is known main pathway of influencing to host cells. ${ }^{22}$ ACE2 is expressed in many tissues, including kidney, bladder and testicular cells in genitourinary system and therefore virus may affect to testicular tissue and cause to testicular tissue damage. ${ }^{23,24}$

As in many medical sections, current literature on the genital involvement of coronavirus is limited with a few case reports. ${ }^{15,16}$ In this case reports, testicular and abdominal pain or both of them continued for eight days. In another case report, a patient with Covid-19 whose complaint was acute abdominal pain diagnosed and revealed ovarian vein thrombosis. Although it develops in a female patient, but this case was significance in terms of genital involvement and it might explained to testicular pain how to occurs by vascular mechanisms. ${ }^{25}$ However pain control was better in our patients of which group 2. Nonsteroidal anti-inflammatory drug therapy was successful in all patients with testicular pain and no patient required more invasive procedures. It can be concluded that this period is shorter than testicular pain due to bacterial 
pathogens. When the patients' history was assessed, having a history of epididymo-orchitis increased the probability of $\mathrm{CoV}$ related testicular pain. Blood-testis barrier defect which is the cause or result of previous epididymo-orchitis; may be one of the reasons for this increase.

In the current population, patients with testicular pain or epididymo-orchitis appeared to be slightly older than expected. Testicular pain or epididymo-orchitis was observed $10.98 \%$ in the study population. One of the most important reasons for this rate to be high is to have a systemic infection condition and it can be explained by the less response to systemic inflammation in the older population, unlike younger men. But this condition was unrelated to the level of lymphopenia. As we mentioned before, the history of previous epididymo-orchitis increases the probability of testicular pain due to $\mathrm{CoV}$. Sun et al. reported that viral RNA was detected in multiple organs in patients with COVID-19 was successfully isolated from urine of patient with COVID-19. ${ }^{26}$ The possibility of having COVID-19 in urine can lead to epididymo-orchitis by vas deferens reflux secondary to increased voiding pressure in aging males.

Another important risk for young adults with testicular pathology is the affecting of male reproductive system $^{27,28}$. This may be a critical reproductive problem for young patients. "Società Italiana di Andrologia e Medicina della Sessualità" and Aversa et al. were reported that in patients recovered from COVID-19, especially for those in reproductive age, andrological consultation and gonadal function evaluation including semen examination should be suggested. ${ }^{29,30}$ Cryopreservation is really important risk for patients in reproductive age due to COVID-19 stored in liquid nitrogen retain its pathogenic properties. ${ }^{31}$ Similar results were reported in the literature for Zika virus which cause to damage on the testicular tissue and male productive system. $^{32,33}$ Therefore, it may be kept in mind in case of a possible pandemic due to Zika virus in the future.

The study has several limitations. In this study, spermiogram and scrotal Doppler ultrasonographic evaluation could not be done in our hospital due to the pandemic; therefore, recommendations were not made to protect the reproductive system of young male patients.

Number of patients in our study is limited; more patients including cohorts or multicentic studies should be done in near future.

\section{Conclusion}

COVID-19 related testicular pain was present more frequently than expected in the study. To explain this frequency and also association of between COVID-19 and testicular pain or epididymo-orchitis; future studies which are isolated the COVID-19 in genitourinary system are necessary.

\section{Ethical Approval:}

This prospective descriptive study was approved by the University of Health Sciences Hamidiye Clinical Research Ethics Committee (Hamidiye-KAEK 20/83).

\section{Patients' Consent:}

Informed consent is not obtained from patients to publish the data concerning this study.

\section{Conflict of Interest:}

Authors declared no conflict of interest.

\section{Authors' Contribution:}

EC: Contributed to the conception and design of the study.

EC, THH, OK, AA, AS: Collected the data.

EC, YO, THH, AS: Drafted and revised the manuscript.

EC, AS, THH, OE: Participated in preparing tables and performing statistical analysis.

\section{References}


1. Borchert A, Baumgarten L, Dalela D, et al. Managing Urology Consultations During COVID-19 Pandemic: Application of a Structured Care Pathway. Urology. 2020.

2. Heldwein FL, Loeb S, Wroclawski ML, et al. A Systematic Review on Guidelines and Recommendations for Urology Standard of Care During the COVID-19 Pandemic. Eur Urol Focus. 2020.

3. Nowroozi A, Amini E. Urology practice in the time of COVID-19. Urol J. 2020;17(3):326.

4. Mejean A, Roupret M, Rozet F, et al. [Recommendations CCAFU on the management of cancers of the urogenital system during an epidemic with Coronavirus COVID-19]. Prog Urol. 2020;30(5):221-231.

5. Cheng Y, Luo R, Wang K, et al. Kidney disease is associated with in-hospital death of patients with COVID-19. Kidney Int.2020;97(5):829-838.

6. Ronco C, Reis T, Husain-Syed F. Management of acute kidney injury in patients with COVID-19. Lancet Respir Med. 2020.

7. Zaorsky NG, Yu JB, McBride SM, et al. Prostate Cancer Radiotherapy Recommendations in Response to COVID-19. Adv Radiat Oncol. 2020.

8. Bhowmick NA, Oft J, Dorff T, et al. COVID-19 and androgen targeted therapy for prostate cancer patients. Endocr Relat Cancer. 2020.

9. Assi T, Ibrahim N, RM KA, et al. The management of patients with metastatic prostate cancer during the COVID-19 pandemic. Future Oncol. 2020.

10. Gomez Rivas J, Dominguez M, Gaya JM, et al. [Prostate cancer and COVID-19 pandemia: Current recommendations.]. Arch Esp Urol.2020;73(5):367-373.

11. de la Pena E, Hernandez V, Guijarro A, et al. [Recommendations on bladder cancer Management during COVID-19 pandemia: lessons learned and future plans.]. Arch Esp Urol. 2020;73(5):374-383.

12. Teoh JYC, Roupret M, Shariat SF, Herrmann T. Intravesical therapy for bladder cancer in the pandemic of Covid-19. World J Urol.2020.

13. Wang T, Liu S, Joseph T, Lyou Y. Managing Bladder Cancer Care during the COVID-19 Pandemic Using a Team-Based Approach. J Clin Med.2020;9(5).

14. Wallis CJD, Novara G, Marandino L, et al. Risks from Deferring Treatment for Genitourinary Cancers: A Collaborative Review to Aid Triage and Management During the COVID-19 Pandemic. Eur Urol.2020.

15. Kim J, Thomsen T, Sell N, Goldsmith AJ. Abdominal and testicular pain: An atypical presentation of COVID-19. Am J Emerg Med. 2020.

16. Gagliardi L, Bertacca C, Centenari C, et al. Orchiepididymitis in a Boy with Covid-19. Pediatr Infect Dis J. 2020.

17. Sigalos JT, Pastuszak AW. Chronic orchialgia: epidemiology, diagnosis and evaluation. Transl Androl Urol. 2017;6(Suppl 1):S37-S43.

18. Ludwig M. Diagnosis and therapy of acute prostatitis, epididymitis and orchitis. Andrologia. 2008;40(2):76-80.

19. Liu JM, Chang YH, Ho TW, et al. Patients with Epididymo-Orchitis and Meteorological Impact in Taiwan: A Nationwide Population-Based Study.Can J Infect Dis Med Microbiol. 2017;2017:1506857.

20. Azmat CE, Vaitla P. Orchitis. In: StatPearls. Treasure Island (FL)2020.

21. Kanda T, Mochida J, Takada S, Hori Y, Yamaguchi K, Takahashi S. Case of mumps orchitis after vaccination. Int $J$ Urol.2014;21(4):426-428. 
22. South AM, Diz DI, Chappell MC. COVID-19, ACE2, and the cardiovascular consequences. Am J Physiol Heart Circ Physiol.2020;318(5):H1084-H1090.

23. Zhang Y, Geng X, Tan Y, et al. New understanding of the damage of SARS-CoV-2 infection outside the respiratory system. Biomed Pharmacother. 2020;127:110195.

24. Fan C, Li K, Ding Y, Lu WL, Wang J. ACE2 expression in kidney and testis may cause kidney and testis damage after 2019-nCoV infection.MedRxiv. 2020.

25. Mohammadi S, Abouzaripour M, Hesam Shariati N, Hesam Shariati MB. Ovarian vein thrombosis after coronavirus disease (COVID-19) infection in a pregnant woman: case report. J Thromb Thrombolysis. 2020.

26. Sun J, Zhu A, Li H, et al. Isolation of infectious SARS-CoV-2 from urine of a COVID-19 patient. Emerg Microbes Infect.2020;9(1):991-993.

27. Illiano E, Trama F, Costantini E. Could COVID-19 have an impact on male fertility? Andrologia. 2020;52(6):e13654.

28. Vishvkarma R, Rajender S. Could SARS-CoV-2 affect male fertility?Andrologia. 2020:e13712.

29. Corona G, Baldi E, Isidori AM, et al. SARS-CoV-2 infection, male fertility and sperm cryopreservation: a position statement of the Italian Society of Andrology and Sexual Medicine (SIAMS) (Societa Italiana di Andrologia e Medicina della Sessualita). J Endocrinol Invest. 2020.

30. Aversa A, Jannini EA. COVID-19, or the triumph of monogamy? Minerva Endocrinol. 2020.

31. De Paoli P. Bio-banking in microbiology: from sample collection to epidemiology, diagnosis and research. FEMS Microbiol Rev.2005;29(5):897-910.

32. Kurscheidt FA, Mesquita CSS, Damke G, et al. Persistence and clinical relevance of Zika virus in the male genital tract. Nat Rev Urol. 2019;16(4):211-230.

33. Ma W, Li S, Ma S, et al. Zika Virus Causes Testis Damage and Leads to Male Infertility in Mice. Cell. 2016;167(6):1511-1524 e1510.

\section{Hosted file}

Tables of COVID-19 Study.pdf available at https://authorea.com/users/356083/articles/483438is-there-any-association-of-covid-19-with-testicular-pain-and-epididymo-orchitis 